\title{
Method to Generate Freeform Surfaces from Idea-sketch and Three Dimensional Data
}

\author{
Tetsuzo Kuragano \\ Meisei University
}

\author{
Susumu Furukawa \\ University of Yamanashi
}

\author{
Mitsuru Kuroda \\ Toyota Technological Institute
}

\begin{abstract}
The Purpose of this study is to generate surface patches which are useful throughout the product-design process in a very short period. We have studied two methods. One is surface generation using a hand-drawn idea-sketch. The other is surface generation using the three dimensional point data measured by commercially obtainable measuring instrument. For hand-drawn idea-sketches, logarithmic transformation, laplacian operation and natural spline curves are introduced. For the three dimensional point data, Delaunay Triangulation is introduced to construct a triangular mesh. Pseudo-normal vectors are calculated using the constructed triangular mesh in a very short time. Productdesigners are able to examine the form of the triangular mesh by shading or newly developed color striped shading using pseudo-normal vectors. Then product designers are able to generate the surface patches which are easy to manipulate for narrowing down to the final design.
\end{abstract}

Key words: freeform surface generation from hand-drawn idea-sketches, light intensity transformation, laplacian operation, natural spline curves, freeform surface generation from three dimensional points, Delaunay Triangulation, Bezier patches, B-spline surfaces

\section{INTRODUCTION}

Conventional design procedures can easily produce simple shapes, but, currently, industrial designers are drawn to aesthetically pleasing freeform shapes because they have great customer appeal, especially in a highly competitive and technically well-developed market, such as that for automobile and electrical appliances. It takes a long time to design aesthetically pleasing product using conventional procedures. 
A solution to this problem is to establish the method to shorten the product design period, especially the period from first-idea generation to narrow down to the final design.

A method capable of producing aesthetically pleasing freeform shapes has these requirements:

- In order to shorten the design period, hand-drawn rendering must be omitted, and a three dimensional real mock-up must be manufactured easily using hand-drawn idea-sketches.

- The form of the hand-made mock-up must be put into the computer in a short time, and the freeform surfaces which are easy to manipulate must be generated using the input data.

- Variation design must be performed using the measured data of a similar product.

To satisfy these requirements we have developed two methods to shorten the idea generation period.

First one is to generate B-spline curves from hand-drawn idea-sketches. Second one is to generate surface patches from three dimensional measured data.

Section 2 of this paper reports a newly developed B-spline curve generation method from hand-drawn idea-sketches, and gives an example of curve generation. Section 3 describes the surface generation method from three dimensional measured data, and gives an example of surface generation.

\section{FREEFORM SURFACE GENERATION FROM HAND -DRAWN IDEA- SKETCHES}

The objective to develop this method is to shorten the product-design period. When designers begin the product design, designers create their ideas and expand them. Normally this process is performed on paper, and designers' hand-drawn lines are called idea-sketches. If the designers' rough idea can be realized as a real mock-up, it will be effective in shortening the design period.

Similar studies on surface generation using idea-sketches have been reported ${ }^{1)}$. Our study does not deal with perspective drawings, therefore our study is not affected by the difference of the drawing method. We have developed a method to extract the freeform curve from the handdrawn idea-sketch which is put into the computer via a scanner. Then we apply the newly developed image processing technique to the digital picture and extract the edges. Extracted edges are converted to B- 
spline curves to make a framework. Then we can generate surfaces from the framework which are extracted from the three-view hand-drawn ideasketches. Using the generated surfaces, we are able to generate the tool paths to machine the mock-ups. Then designers are able to examine the mock-ups visually and tactually.

In this manner designers are able to acquire three dimensional machined mock-ups easily from the idea-sketch. The key to this method is that precision is not required or needed, but the design period is shortened remarkably.

\subsection{Light intensity transformation}

Generally, various types of gradation are painted as a background in the hand-drawn idea-sketches ${ }^{2}$. In such cases, human eyes are able to recognize lines which are drawn in the dark area as well as lines which are drawn in the bright area.

But the computer can not recognize lines in the dark area, because of the small difference of the light intensity between the line and gradation. Therefore we have developed a method to extract lines which are drawn in the relatively dark area. Original digital picture is transformed into the logarithmic like picture.

This means that in the transformed picture the light intensity difference of adjacent picture element in the dark area becomes very close to the difference in the bright area. In other words, the light intensity of the dark area of the original picture can be amplified, while that of the bright area of the original picture is reduced.

By transforming the original picture into logarithmic like picture, we are able to extract the lines applying the same method without considering the value of the light intensity.

\subsection{Threshold determination}

Histogram is a graph which shows the number of frequency of the gray level. When the image signal is analog, this histogram becomes a curve, but when digital, histogram becomes a bar graph, denoting horizontal axis as light intensity and vertical axis as frequency. In case the histogram has two peaks (ridges), the valley part may be threshold.

But two peaks suggest the possibility of two edges existence. In other words, there may exist edges in the relatively dark area and in the relatively bright area. If the valley part is decided as a threshold, we will lose the opportunity to extract the edge in the relatively dark area. In general, designers use various types of gradation in their idea-sketches, so there 
might be many peaks in the histogram. Therefore, the threshold determination should be performed interactively.

Conversion to binary picture should be performed according to the threshold temporarily decided. Then, the candidate points for edges are displayed. This determination process should be done by the designers.

\subsection{Edge detection}

Second derivative in the image processing study is called laplacian. Applying the laplacian window to the digital picture, the position of the edge in the picture is detected. This position is corresponding to the position of the line of the original picture in which we can see lines of the hand-drawn idea-sketch. An example of the edge detection is shown in Figure 1. This shows that the idea described in sub-section 2.1 is working effectively.

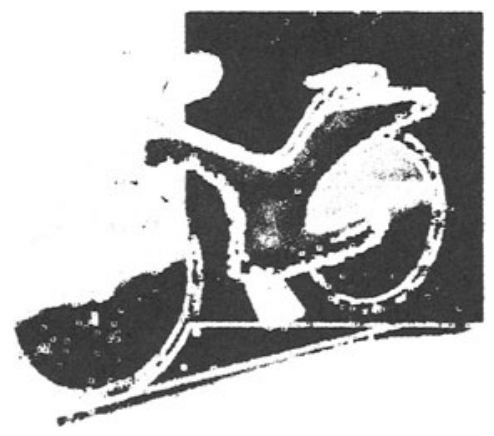

Figure 1. An example of edge detection

\subsection{Natural spline curve generation from the edge}

In this sub-section, natural spline curve generation from binary edges is described. Binary edges are recognized as discrete positional data. Using these positional data, natural spline curves are generated. According to the book written in Japanese by A.Sakurai, natural spline curve which smoothens the given points are proposed by Schoenberg ${ }^{3)}$. We use A.Sakurai's method to derive the natural spline function which is described in his book. His natural spline function depends on the coordinate system. So we parametrize his natural spline function and we express $(2 M-1)$ degree natural spline curve as

$$
\mathbf{C}(t)=\mathbf{P}_{M-1}(t)+\sum_{i=0}^{N-1} \mathbf{b}_{i}\left(t-t_{i}\right)_{+}^{2 M-1}
$$


where $\quad \mathbf{P}_{M-1}(t)=\sum_{i=0}^{M-1} \mathbf{a}_{i} t^{i}$

For $\mathbf{C}(t)$ is a $(2 M-1)$ degree natural spline function and smoothest for the given points, equation (2) and (3) should be satisfied simultaneously.

$$
\begin{aligned}
& \sum_{i=0}^{N-1} \mathbf{b}_{i} \cdot t_{i}^{r}=\mathbf{0} \\
& (r=0,1, \cdots, M-1) \\
& \mathbf{C}\left(t_{j}\right)+(-1)^{M} g(2 M-1) ! \mathbf{b}_{j} w_{j}^{-1}=\mathbf{Q}_{j} \\
& (j=0,1, \cdots, N-1)
\end{aligned}
$$

where $\mathrm{g}$ is positive constant and $w_{j}$ is positive weight. $N$ is the number of given points and $\mathbf{Q}_{j}$ is positional vector of given points. Solving $\mathbf{a}_{i}(i=0,1, \cdots, M-1)$ and $\mathbf{b}_{j}(j=0,1, \cdots, N-1)$, natural spline curve is defined using equation (1). Generated natural spline curves are converted to the non uniform B-spline curve.

\subsection{An example of surface generation from hand-drawn idea-sketch}

Using the method mentioned in the previous sub-sections, we describe the freeform surface generation process from hand-drawn idea-sketches for automobile body shell design according to the following generation steps.

1) Figure 2 shows the hand-drawn idea-sketch of the side-view of the automobile. This sketch is put into the computer via a scanner. Then the idea-sketch is displayed on the computer monitor.

2) We indicate the area in which the edges are extracted by defining the multi-sided polygon. Then the candidate points for the edge are calculated. Unneeded points are eliminated interactively according to the designer's intention. The result is shown in Figure 3.

3) Natural spline curve is generated using the points after elimination.

Natural spline curve is replaced by non uniform B-spline curve. Radius of curvature of the generated curve are displayed with the idea-sketch. Those are shown in Figure 4.

4) Repeating this operation, curves which are necessary for product design 
can be generated from this side-view. For the front-view and top-view the same operation is performed. Side-view, front-view, and top-view are located in the three dimensional space. We sweep the lines on each view perpendicular to corresponding view, then ruled surfaces are generated. Taking the intersection of these ruled surfaces, then special curves which may be a framework of the surface can be calculated. Repeating these operation, a framework is generated in the three dimensional space.

Generated framework is shown in Figure 5.

5) Interpolating the framework, freeform surfaces are generated. These surfaces are shown as a shading display in Figure 6.

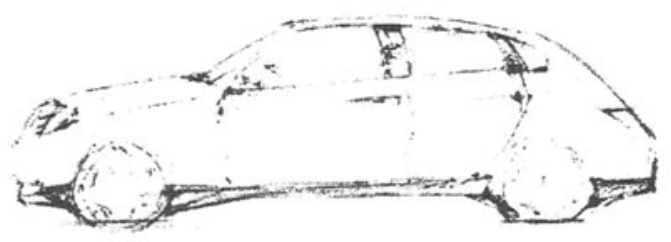

Figure 2. Hand-drawn idea-sketch

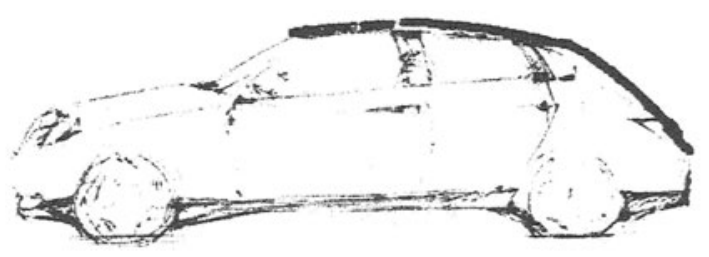

Figure 3. Unneeded points are eliminated.

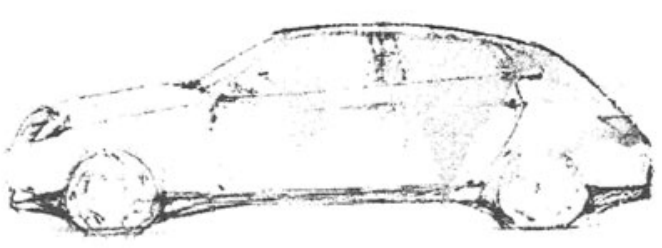

Figure 4. Concerted to B-spline curve 


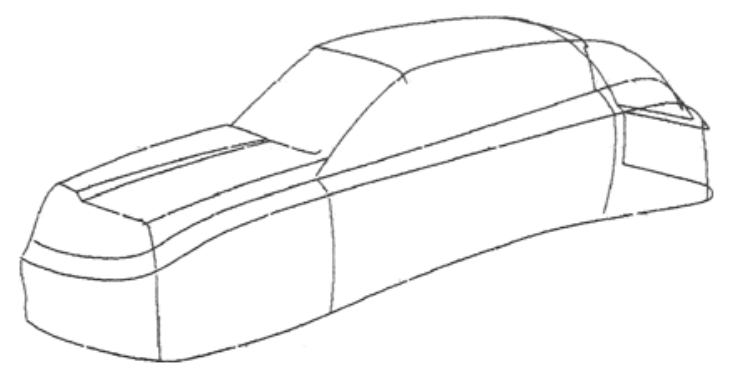

Figure 5. Generated framework

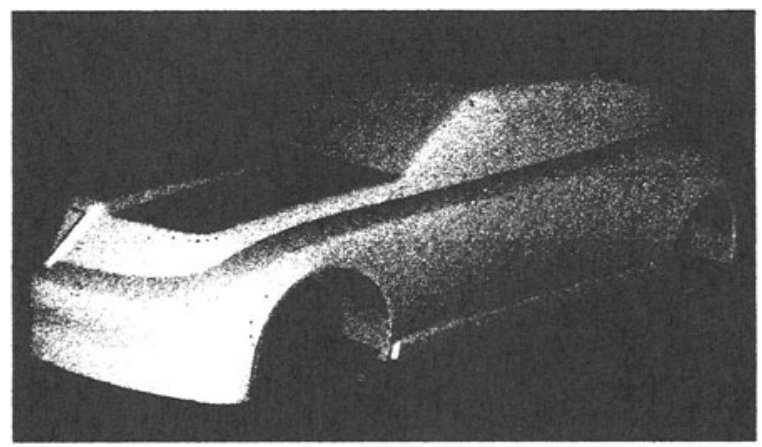

Figure 6. Shaded picture of generated surfaces

\section{FREEFORM SURFACE GENERATION FROM THREE DIMENSIONAL MEASURED DATA}

The objective of this study is to develop a method to generate freeform surfaces which are useful in the product-design process. To put freeform shape into the computer by using keyboard, mouse, and dial, while watching the computer monitor, it takes a long time and a lot of energy, and skills are needed. To avoid these, a method to generate freeform surfaces from three dimensional measured data is commonly used. In addition to time and labor saving, there are two designers' requirements.

1) The form data of similar products must be put into the computer, so that designers will be able to perform the variation design.

2) The boundary of the patch or the surface must be determined so that these can be easily modified throughout the product-design process. 
Many studies on surface generation using three dimensional data have been reported ${ }^{4) 5}$ ). In this study the object to be measured is normally a mock-up which is manufactured during the product-design process, therefore it is not a master model, but a mock-up close to the final form.

Also, in this study, easy manipulation of surface patches is strongly considered when surface patches are generated from the three dimensional data. Normally three dimensional data are measured without considering the surface boundary.

We introduced Delaunay Triangulation to construct a triangular mesh. If a triangular mesh is not constructed, it will take a long computation time to get pseudo-normal vectors at all the measured points.

\subsection{Conventional method}

At the design studio where one of the authors of this paper work, three dimensional data was measured tactually and manually, while indicating the characteristic part by the colored tape. The problem of this method is that it forces the human to indicate the characteristic part of the three dimensional object according to their expertise. In addition to that he has to spend a long time measuring the object. More over the measuring probe is moved and located manually, therefore measuring errors will become relatively large and the smoothening operation of the curve takes a long time.

Another method we have tried is to measure the three dimensional object in a lattice-like manner. Then, we generate the B-spline surface through the given measured points. This method is very easy to decide the measuring direction and measuring pitch, and to generate the B-spline surfaces. But measuring position are decided without considering the feature of the three dimensional object. Therefore at a certain part, because of the lack of measuring points, we cannot reconstruct the object. On the other hand, if we make the measuring pitch narrower, surface modification time becomes longer, more over unintentional wobbling of the surface will occur because of the knot vector, since B-spline surface uses bivariate blending function and only two knot vectors are available for $\mathrm{u}$ and $\mathrm{v}$ direction.

In addition to this, the generated surfaces include the errors while measuring, since there is no way to know whether measured data includes errors or not.

\subsection{Pseudo-normal vector calculation}

Assume the number of vertices of the triangular mesh which is the number of the measured point be $n$, pseudo-normal vector $\mathbf{N}_{i}$ at each measured vertex $\mathbf{P}_{i}(1 \leq i \leq n)$ can be calculated by taking the mean value 
of $m$ triangle normal vector $\mathbf{Q}_{i}(1 \leq i \leq m)$. This is shown in Figure 7 . Doing the same operation at all the vertices, we can calculate the pseudonormal vectors which correspond to the measured points.

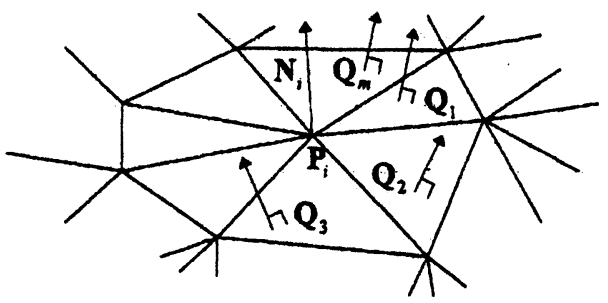

Figure 7. Pseudo-normal vector calculation

\subsection{Triangle edge replacement by Bezier curve}

Each edge of the triangle which is one element of the triangular mesh is replaced by the cubic Bezier curve. Cubic Bezier curve is represented by the equation (4) ${ }^{6}$. Both sides of the Bezier curve control point $\mathbf{P}_{0}$ and $\mathbf{P}_{3}$ are the vertices of the triangle. Inner control points are $\mathbf{P}_{1}$ and $\mathbf{P}_{2}$. Calculation method for $\mathbf{P}_{1}$ is shown by the equation (5) and (6). Control point $\mathbf{P}_{2}$ is able to be calculated in the same manner.

$$
\mathbf{C}(u)=(1-u+u E)^{3} \mathbf{P}_{0}
$$

where $u$ is a parameter which varies from 0 to $1 . E$ is a shifting operator and has the following relation.

$$
\begin{aligned}
& \mathbf{P}_{i+1}=E \cdot \mathbf{P}_{i} \\
& \mathbf{a}=\left(P_{i} P_{a 1} \times \mathbf{N}_{i}\right) \times \mathbf{N}_{i} \\
& \mathbf{P}_{1}=\mathbf{P}_{0}+\frac{\mathbf{a}}{|\mathbf{a}|} \cdot \frac{\left|P_{i} P_{a 1}\right|}{3}
\end{aligned}
$$

Each edge of the triangle of the triangular mesh is able to be replaced by the cubic Bezier curves applying the above mentioned method. This method is illustrated in Figure 8(a). Figure 8(b) is illustrated two dimensionally to understand the replacing method easily. Replacing the edges by the cubic Bezier curve using the above mentioned method, all Bezier curves are tangential plane continuous at $\mathbf{P}_{i}$. The normal vectors of the tangential plane at $\mathbf{P}_{i}$ is equal to the pseudo normal vector $\mathbf{N}_{i}$. In the case when measured points are scatteredly located, if we apply the above method, we can interpolate the points close to the real form rather than the straight line. The reason why we decide the cubic Bezier curve is that it is easy to calculate the inner two control points. 


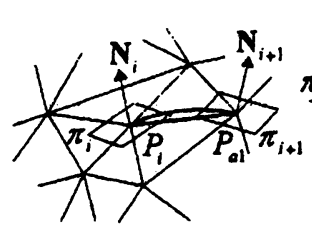

(a)

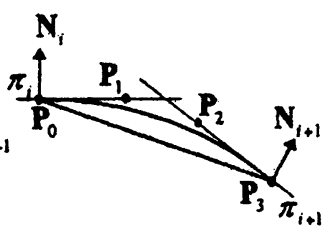

(b)

Figure 8. Replacing triangle edges by Bezier curves

\subsection{Triangular mesh visualization}

The measured points show the form vaguely. But to examine the form which is constructed by the measured point data, we have developed the following visualization technique.

- Shading display: using the vertex $(x, y, z)$ values and corresponding pseudo-normal vector values, we can calculate the light intensity, and interpolate the inner domain of the triangle, we can display the shaded picture of three

dimensional objects.

- Color striped shading: Converting the variation of the light intensity to the variation of the color combining the three primary colors of red, green and blue, we are able to display the color striped shading. This method is useful to examine the highlighted line location.

An example of the color striped shading display is shown in Figure 9.

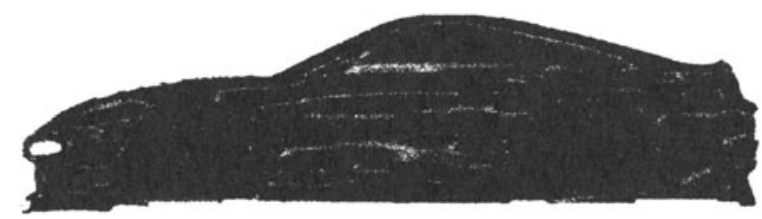

Figure 9. Color striped shading display

\subsection{Large angle variation point extraction}

Choosing 2 vertices out of 3 vertices of the triangular mesh, and calculating the 2 pseudo-normal vectors $\mathbf{N}_{i}, \mathbf{N}_{j}$ which correspond to the vertices, if $\mathbf{N}_{i}, \mathbf{N}_{j}$ satisfy the equation (7), while $\theta_{3}$ is determined by the designers, $\mathbf{P}_{i}$ and $\mathbf{P}_{j}$ can be candidate points which show large angle variation. Using this method, we are able to extract the points which may 
represent the feature (characteristic part) of the three dimensional object. Since features have steep normal vector variation normally. From these points, we are able to generate Bezier curve or B-spline curve by applying the least square method. Then we can generate the curve as a boundary or as a trimmed boundary of patches or surfaces.

An example of extracted points is shown in Figure 10.

$$
\cos \theta_{3} \leq \frac{\mathbf{N}_{i} \cdot \mathbf{N}_{j}}{\left|\mathbf{N}_{i}\right| \cdot\left|\mathbf{N}_{j}\right|}
$$

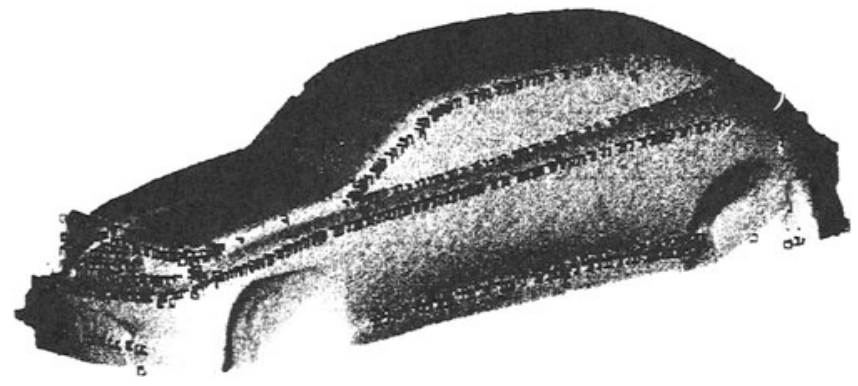

Figure 10. Large angle variation extraction

\subsection{Patch boundary determination and patch generation}

For generating Bezier surface patches, to generate a Bezier curve boundary is needed. For this purpose a triangular mesh whose edges are replaced by cubic Bezier curves are cut by the plane or Bezier surface patch. Then intersection points are used to generate the curve by using the least square method. This process automatically smoothens the measured point data. Repeating this, four boundaries of a Bezier patch are determined. Using the least square method, four inner control points of the Bezier patch are determined. Doing these operations, we are able to generate very smooth surface patches.

\subsection{An example of surface generation using three dimensional measured points}

We have generated freeform surface by measuring the clay model of the automobile whose scale is approximately one fifth of real size for a trial.

Using the technique described in the previous sub-Sections, we have generated the automobile body shell. We describe the surface generation process according to the following generation step. 
1) Measured point data are shown in Figure 11. Darker parts show that the measured points are overlapped.

2) Applying Delaunay Triangulation, we have constructed triangular meshes. These meshes are shown in Figure 12. We have changed the color of these mesh to identify the measured data set. Figure 13 shows the close up of the part indicated by the arrow in Figure 12. So we can see the triangular mesh. 3) Applying the shading method to the triangular mesh, we can examine the three dimensional object. At the same time we can find out the error of the measured data. This is shown in Figure 14. We can see the bumps around the wind-shield of the automobile. These show the errors of measured data.

4) Color striped shading is applied to the triangular mesh. This is shown in Figure 15. We can examine the highlighted line which may be a characteristic line of the object.

5) Applying the above mentioned procedure, we can decide the surface boundary from the triangular mesh. Then, we can generate surfaces. These are shown in Figure 16.

6) Using the shading and texture mapping technique we have developed, we can display our computer generated rendering picture. This is shown in Figure 17.

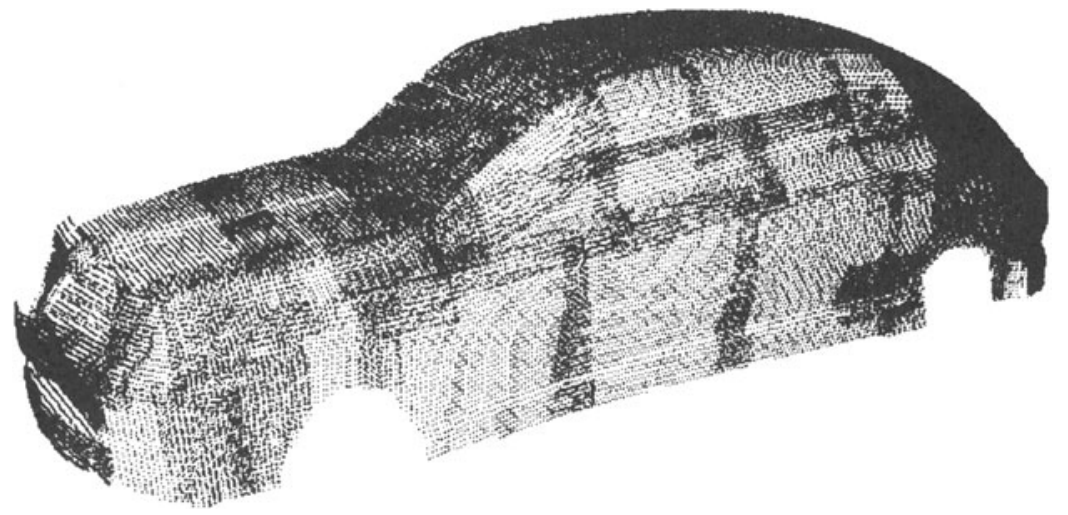

Figure 11. Measured point data 


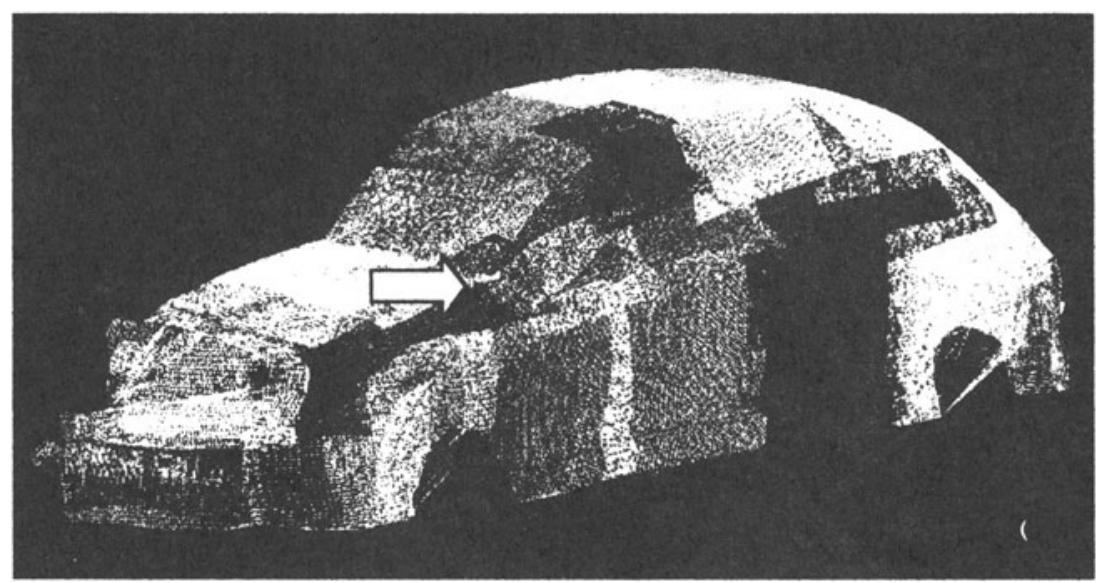

Figure 12. Triangular mesh

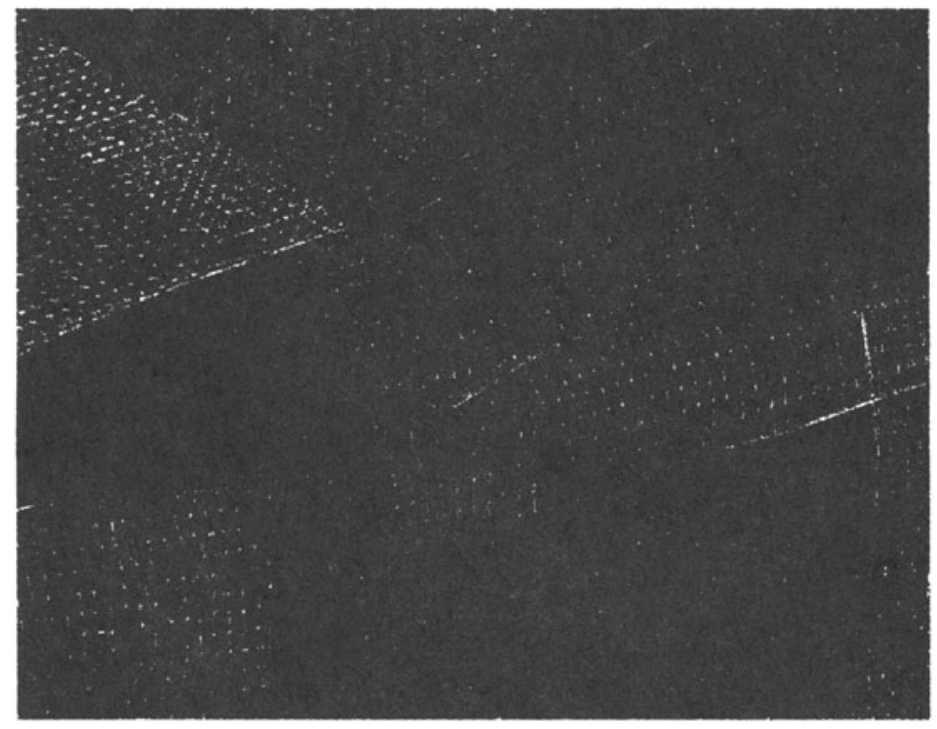

Figure 13. Close -up of triangular mesh 


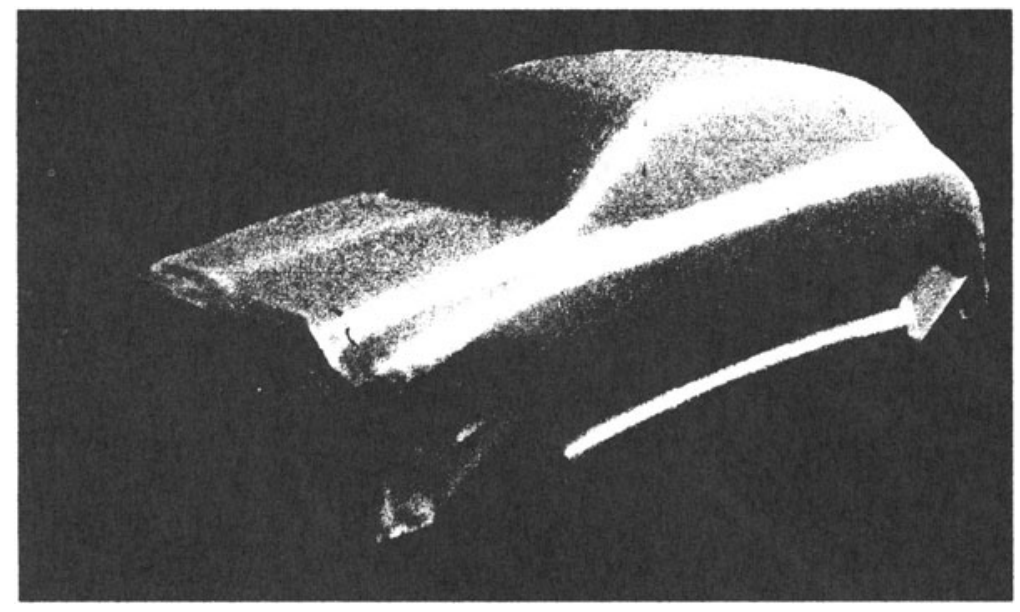

Figure 14. Shaded picture of triangular mesh

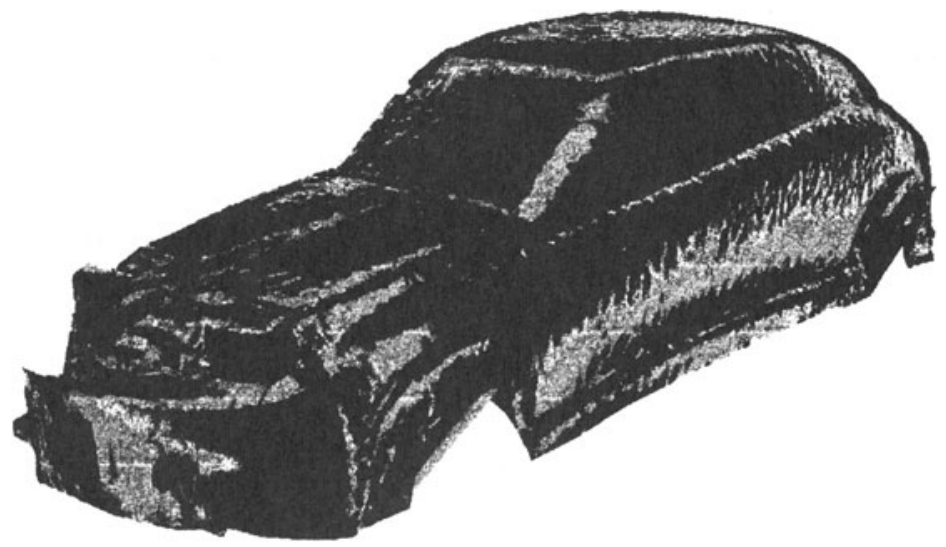

Figure 15. Color striped shading display of triangular mesh 


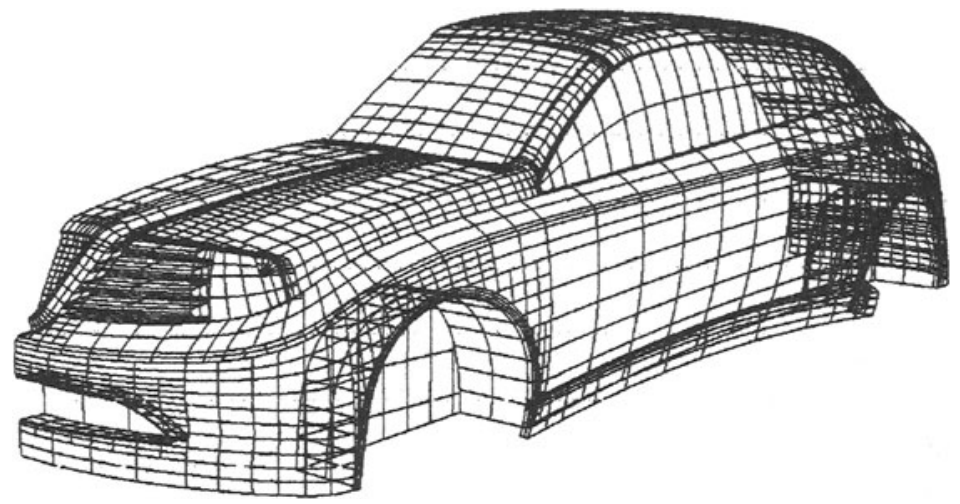

Figure 16. Framework of the generated surfaces

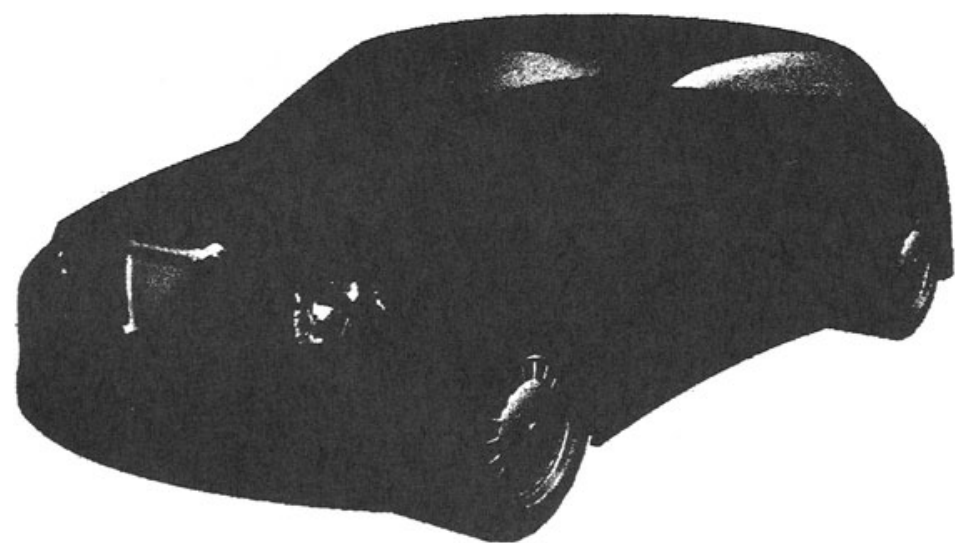

Figure 17. Shading with texture mapped surface

\section{CONCLUSION}

We have developed two methods to shorten the product design period. These methods have the following features.

1) Using a newly developed image processing technique, designers are able to extract edges located even in the relatively dark area, then generate natural spline curve to get non uniform B-spline curve.

2) Constructing a triangular mesh from measured point data, designers are able to examine the form by shading or color striped shading. Also they are 
able to extract the point data which may construct characteristic lines. Then designers are able to generate the freeform curve using extracted points as a patch boundary or surface trimmed boundary. These patches and surfaces are easily manipulated throughout the product-design process.

Using these two methods designers are able to generate freeform surfaces from hand-drawn idea-sketches or hand-made mock-ups, and examine the form by the various kind of display techniques, then they are able to obtain the freeform surfaces as a real mock-up. Also designers are able to use the similar form of different products for variation design.

Using these two methods, productivity of the product-design improved remarkably.

\section{REFERENCES}

I. Furushima,S., Kanai,S., Takahashi,H., " Generation of 3 Dimensional Free-form Curve Model by Automatic Recognition of Rough Sketch", JSPE59-06'93-06-969.(1993)

2. Ungar,J., "Rendering in Mixed Media", (Watson-Guptill Publications, 1985).

3. Schoenberg,I.J., "Spline functions and the problem of graduation", Proceedings of the National Academy of Science of the U.S.A., 52, 947-950. (1964)

4. Oblonsek,C., and Guid,N., "A Fast Surface Based Procedure for Object Reconstruction from 3 D Scattered Points", Computer Vision and Image Understanding, Vol.69, No2 185-195. (1998)

5. Dietz, U., "Fair Surface Reconstruction from Point Clouds", Mathematical Methods for Curves Surfaces II, 79-86. (1998)

6. Hosaka, M., "Modeling of Curves and Surfaces in CAD/CAM", (Springer-Verlag, 1991). 